\title{
Effects of clay mineralogy and physico-chemical properties on potassium availability under soil aquic conditions
}

\author{
A. Raheb ${ }^{1 *}$ and A. Heidari ${ }^{1}$ \\ ${ }^{I}$ Soil Science Department, Faculty of Agricultural Engineering and Technology, College of Agriculture and \\ Natural Resources, University of Tehran, Karaj, Iran. ${ }^{*}$ Corresponding author: ahaidari@ut.ac.ir
}

\begin{abstract}
Aquic conditions performed for paddy cultivation may cause many nutritional problems. Clay mineralogical composition plays an important role in this relation by affecting many physico-chemical and biological characteristics of soils. Three forms of $\mathrm{K}$ including unavailable, slowly available and readily available $\mathrm{K}$, exist in equilibrium in the soil system. Relationships between soil potassium forms with the clay mineralogical suite and physico-chemical properties were determined in 33 soil samples collected from five from paddy soils, two from kiwi fruit and one from citrus land pedons in the North of Iran. Mineralogical analyses showed that smectite, illite and vermiculite were the most abundant clay minerals in the studied soils. Mean soluble and exchangeable potassium in paddy soils were rather low compared to non-paddy soils due to potassium fertilization in non-paddy soils. Non-exchangeable potassium in the samples containing smectite as dominant clay mineral was lower than the samples dominated by vermiculite, hydroxy-interlayer vermiculite (HIV) and illite. Inability of the studied soils for potassium fixation was related to prevailed montmorillonitic type of smectite, which its layer charge originates from octahedral sheet. Significant correlations $\left(0.56^{* *}, 0.54^{* *}, 0.56^{* *}\right)$ were obtained between exchangeable potassium and clay content, organic carbon and cation exchange capacity respectively. Therefore, taking into account both exchangeable and non-exchangeable $\mathrm{K}$ beside clay mineralogy and physico-chemical properties gives better indications for $\mathrm{K}$ potential and soil-quality management, compared to exchangeable form of $\mathrm{K}$ alone.
\end{abstract}

Keywords: Exchangeable K, Kiwi, Mineralogy, Non-exchangeable K; Paddy soil, Smectite, Vermiculite. 


\section{Introduction}

Longtime ponding of soil in the wake of rice growing and the resulting oxygen-free environment finally cause aquic soil moisture regimes or aquic soil conditions. These affect soil quality, plant growth and desired conditions for other soil living organisms (Soil Survey Staff, 2010).

Worldwide, the total rice cultivated area covers approximately 150 million hectares, of which approximately 90\% are situated in Asia (Witt and Haefele, 2005). Climate and landforms are the two determinants factors for a subsequent strongly biased distribution of paddy lands and their fertility (Kyuma, 2004). The subsoil, in well drained soils containing a perched water table over an impermeable layer near surface, such as the plow pan in rice fields can be aerobic; while in the throughout poorly drained soils, the subsoil is normally anaerobic. The subsoil may provide significant quantities of nutrients to plants growing in the soil if their roots can reach there (Witt and Haefele, 2005). Rice cultivation has great importance in the North of Iran, especially in Mazandaran and Guilan provinces which comprise $78 \%$ of rice cultivation extent and yield of the whole country (Rahimi Petroudi et al., 2011).

Potassium is an essential and major nutrient for crop production (Alfaro et al., 2003; Zhang et al., 2011). Presently, paddy farmers either do not use $K$ fertilizer or the used amount is insufficient to balance $\mathrm{K}$ removal with grain and straw (Witt and Haefele, 2005). Soil potassium is classified to three forms according to its availability: unavailable, slowly available and readily available or exchangeable (Sharpley, 1989; Bhonsle et al., 1992). Unavailable form comprises approximately $90-98 \%$ of total soil K (Sparks,
1987). Slowly available (non-exchangeable) $\mathrm{K}$, is a form $\mathrm{K}$ that has trapped between the layers or sheets of certain kinds of clay minerals and plants can use only very little of it during a single growing season. The major sources of non-exchangeable $\mathrm{K}$ in soils are K-rich 2:1 clay minerals such as illites and vermiculites (Sparks, 1987; Moritsuka et al., 2004; Britzke et al., 2012). Depending on their weathering stage and source of electrical charge; $\mathrm{K}$ release from the interlayer space of such minerals is very slow, (Barré et al., 2007).

The release of $\mathrm{K}$ from clay minerals is influenced by particle size distribution and chemical composition (Huang, 2005). Different articles have reported that plants can take up both exchangeable and nonexchangeable forms of potassium (Ghosh and Singh, 2001; Towfighi, 1998). Readily available potassium is a dissolved form of $\mathrm{K}$ (water-soluble) or $\mathrm{K}$ held on the outer surface of clay particles (exchangeable K). Plants can take up both exchangeable and non-exchangeable forms of potassium. Bhonsle et al. (1992) suggested that fixed or non-exchangeable forms of $\mathrm{K}$ can even be the main source of potassium for plants. Minerals' $\mathrm{K}$ release to soluble and exchangeable forms and its adsorption by exchange sites depends on the equilibrium between different phases of soil $\mathrm{K}$, which may be affected by such factors as root uptake, applied fertilizer $\mathrm{K}$, soil moisture, soil $\mathrm{pH}$ and soil temperature (Zhang et al., 2011; Britzke et al., 2012).

The relationship between clay mineralogy composition and potassium forms and physico-chemical properties has been demonstrated by several studies (Sharply, 1989; Bhonsle et al., 1992; Ghosh and Singh, 2001; Surapaneni et al., 2002; Srinivasarao 
et al., 2006). Relationships between clay mineralogy and potassium forms can be used in evaluating potential soil $\mathrm{K}$ fertility, prediction of $\mathrm{K}$ cycling and plant uptake (Sharply, 1989). Information on the $\mathrm{NH}_{4} \mathrm{OAc}$ $\mathrm{K}$ form of potassium (i.e. soluble and exchangeable $\mathrm{K})$ along with knowledge of mineralogical clay composition can provide insights into the equilibrium and release of non-exchangeable $\mathrm{K}$ to plants and the need for K fertilizers (Bhonsle et al., 1992).

Soils with high content of 2:1 clay minerals (micas, vermiculite and high-layer-charge smectite) contain larger amounts of non-exchangeable $\mathrm{K}$ than those with kaolinite and other siliceous minerals (Sharply, 1989; Ghosh and Singh, 2001). Bhonsle et al. (1992) stated that while kaolinitic soils had low levels of $\mathrm{NH}_{4} \mathrm{OAc}-\mathrm{K}$, mixed and illitic soils had medium levels and smectitic soils had high levels; their estimates for $\mathrm{HNO}_{3}-\mathrm{K}$ (non-exchangeable $\mathrm{K}$ ) showed that kaolinitic, mixed and smectitic soils had low, and illitic soils had high levels. Their study set out the proportion of $\mathrm{NH}_{4} \mathrm{OAc}-\mathrm{K}$ to $\mathrm{HNO}_{3}-\mathrm{K}$ in the sequence smectitic $>$ kaolinitic $>$ mixed $>$ illitic. Sharpley (1989) stated that determining both exchangeable and non-exchangeable K could give a better indication of a soil's potential K-supplying power than only of exchangeable form.

There is no report on the clay mineralogy and $\mathrm{K}$ status of the land used for different agricultural purposes in northern Iran (although, Towfighi (1998) estimated that available $\mathrm{K}$ in more than 100,000 ha of Iranian paddy soils varies between lower-than-critical to moderate). Taking into account that $\mathrm{K}$ is an essential plant nutrient especially for rice cultivation. Increasing $\mathrm{K}$ deficiency due to longtime cultivation without its supplying has been reported for paddy soils around the world. Therefore, the objectives of the present study are: (1) to compare different forms of potassium (soluble $\mathrm{K}$, exchangeable $\mathrm{K}$ and non-exchangeable $\mathrm{K}$ ) in paddy soils and non-paddy soils, and (2) to compare the relationships between forms of $\mathrm{K}$ with physicochemical properties and clay mineralogy in paddy soils, and soils under citrus and kiwi fruit cultivation.

\section{Materials and Methods}

\subsection{Study Region and Field Sampling}

This study examined paddy and non-paddy soils (about 10 ha) obtained from the Rice Research Institute of Iran ( $36^{\circ} 51^{\prime} 53^{\prime \prime}$ to $36^{\circ} 51^{\prime} 48^{\prime \prime}$ northern latitudes and $50^{\circ} 46^{\prime} 56^{\prime \prime}$ to $50^{\circ} 46^{\prime} 44^{\prime \prime}$ eastern longitudes, $20 \mathrm{~m}$ below sea level) (Figure 1). Mean annual precipitation in this region is $1253 \mathrm{~mm}$, and mean annual air temperature is $15.8{ }^{\circ} \mathrm{C}$. Soil moisture and temperature regimes, calculated according to the Newhall Simulation Model (Newhall and Berdanier, 1996), were udic and thermic, respectively. Three adjacent land uses, including paddy, kiwi fruit and citrus were selected. The selected area has high water table (depth $<50 \mathrm{~cm})$ in most parts and also is waterlogged in the paddy cultivated pedons. Thus it has specific conditions with underneath and surface saturation. Eight pedons in total were dug, described and sampled according to the field book for describing and sampling soils, and were classified according to the Soil Taxonomy (Soil Survey Staff, 2010). From the pedon samples, five pedons from paddy soils (with high water table and surface ponding), two pedons from kiwi fruit (just pedon 7 contains aquic condition) and one pedon from citrus (non aquic) were included for comprehensive analysis in this study. 


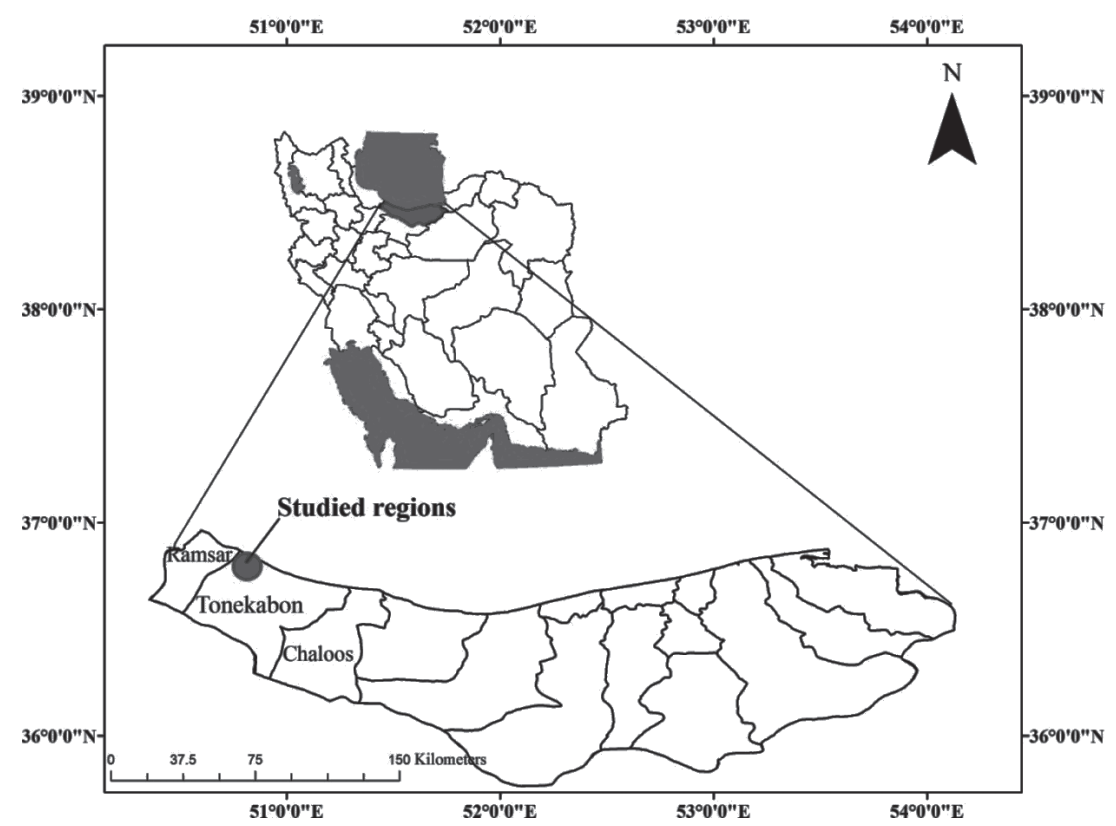

Figure 1. Map of studied region in Mazandaran province, northern Iran.

\subsection{Physico-Chemical Analysis}

All analyses were performed on air-dried soil samples passed through a $2 \mathrm{~mm}$ sieve (Pansu and Gautheyrou, 2006). The samples' physico-chemical and mineralogical characteristics were analyzed according to standard methods (Kunze and Dixon, 1986). $\mathrm{pH}_{\mathrm{sp}}$ was determined using a electrical $\mathrm{pH}$ meter applied to saturated soil paste using deionised water. Electrical conductivity $\left(\mathrm{EC}_{\mathrm{se}}\right)$ was measured in the soil saturated extract (Carter and Gregorich, 2008). Particle-size distribution (PSD) was measured by the hydrometer method. Organic carbon (OC) was determined using wet oxidation (Pansu and Gautheyrou, 2006). Cation exchange capacity (CEC) was determined by the ammonium acetate method $(\mathrm{pH}=7)$ (Carter and Gregorich, 2008). Calcium carbonate equivalent (CCE) was determined for some soil samples by acid neutralization (Carter and Gregorich, 2008). Water soluble $\mathrm{K}$ was measured in the saturated extract using flame photometer. Exchangeable potassium was determined by shaking $2.5 \mathrm{~g}$ air-dried soil with $50 \mathrm{~mL}$ of 1 $\mathrm{M} \mathrm{NH}_{4} \mathrm{OAc}$ solution ( $\mathrm{pH}=7$ ) (Knudsen et al., 1996). Non-exchangeable potassium was determined by heating $10 \mathrm{~g}$ of soil with $25 \mathrm{~mL}$ of $1 \mathrm{M} \mathrm{HNO}_{3}$ solution in an oil bath $\left(113^{\circ} \mathrm{C}\right)$ for 25 minutes and subtracting the sum of soluble and exchangeable forms (Pratt, 1965). The extracts' $\mathrm{K}$ concentrations were determined by flame photometry.

\subsection{Mineralogical Analysis}

Clay mineralogical studies were performed by removing soluble salts and gypsum by washing out (Konse and Rich, 1959), carbonates by neutralization with sodium acetate $(\mathrm{pH}=5)$ (Grossman and Millet, 1961), organic materials by oxidation with $\mathrm{H}_{2} \mathrm{O}_{2}$ (Konse and Rich, 1959) and ferrous oxides by citrate dithionate bicarbonate (Mehra and Jackson, 1960). Clay fraction was separated by sedimenta- 
tion and saturated with $\mathrm{Mg}^{2+}$ and $\mathrm{K}^{+}$ions using 1 $\mathrm{M} \mathrm{MgCl}_{2}$ and $\mathrm{KCl}$ solutions. Mineralogical composition was determined by X-ray diffraction using a Siemens D5000 diffractometer via $\mathrm{CuK} \alpha(\lambda=1.5409$ $\AA)$ and $30 \mathrm{kV}$ voltage and $30 \mathrm{~mA}$. Clay mineralogical composition was determined using the intensity and position of the X-ray diffractogram peaks, considering clay apparent $\mathrm{CEC}$ (CEC/Clay \%). Considering an average of $200 \mathrm{cmol}^{+} \mathrm{kg}^{-1} \mathrm{CEC}$ for soil organic matter and multiplying it by organic matter content the apparent clay CEC was obtained from subtracting organic matter CEC from measured CEC. The ratio of CEC by ammonium acetate at $\mathrm{pH} 7$ to clay percent can be used to estimate clay mineralogy and clay dispersion. If the ratio is multiplied by 100 , the product is $\mathrm{cmol}^{+} \mathrm{kg}^{-1}$ clay. The following ratios are typical for the following classes of clay mineralogy: less than 0.2 , kaolinitic; $0.2-0.3$, kaolinitic or mixed; 0.3-0.5, mixed or illitic; 0.50.7 , mixed or smectitic; and more than 0.7 , smectitic (Soil Survey Staff, 2010).

\subsection{Statistical Analysis}

Statistical analysis was performed using SPSS 17.0 software to examine the correlations between the results and their dependency.

\section{Results}

The paddy soils showed very distinct morphological, physical and chemical properties from those of nonpaddy soils. Table 1 and Table 2 shows some morphological and physico-chemical characteristics of the studied pedons, including $\mathrm{pH}, \mathrm{EC}, \mathrm{CEC}, \mathrm{OC}, \mathrm{PSD}$ and different forms of potassium.

Table 1. Some morphological properties of the studied pedons.

\begin{tabular}{|c|c|c|c|c|c|c|}
\hline Horizon & Depth & Structure $^{1}$ & Void $^{2}$ & $\operatorname{Root}^{3}$ & Color & $\begin{array}{c}\text { Redoximorphic } \\
\text { properties }^{4}\end{array}$ \\
\hline \multicolumn{7}{|c|}{ P1- Citrus $\left(36^{\circ} 51^{\prime} 52.3^{\prime \prime} \mathrm{N} 50^{\circ} 46^{\prime} 55.4^{\prime \prime} \mathrm{E}\right)$} \\
\hline Ap & $0-13$ & Massive & $1 \mathrm{~F}+\mathrm{VFI}$ & $3 \mathrm{~F}+\mathrm{M}+\mathrm{C}$ & $10 Y R 3 / 2$ & - \\
\hline $\mathrm{AC}$ & $13-23$ & $\mathrm{Sg}$ & $1 \mathrm{~F}+\mathrm{VF}$ & $2 \mathrm{~F}+\mathrm{M}$ & 10YR 3/6 & - \\
\hline $\mathrm{C} 1$ & $23-61$ & $\mathrm{Sg}$ & $1 \mathrm{~F}+\mathrm{VFI}$ & $1 \mathrm{~F}+\mathrm{M}$ & $5 Y R 3 / 4$ & - \\
\hline $\mathrm{C} 2$ & $61-100$ & $\mathrm{Sg}$ & $1 \mathrm{VFI}$ & - & 10YR $3 / 3$ & Gleying \\
\hline \multicolumn{7}{|c|}{ P2- Paddy $\left(36^{\circ} 51^{\prime} 50.4^{\prime \prime}\right.$ N 50 46 52.6" E) } \\
\hline Apg & $0-20$ & Massive & $1 \mathrm{VFI}$ & $3 \mathrm{~F}+\mathrm{VF}$ & $2.5 \mathrm{Y} 4 / 2$ & - \\
\hline $\mathrm{Bg}$ & $20-38$ & $\begin{array}{l}2 \mathrm{~m} \mathrm{abk} \\
2 \mathrm{~m} \mathrm{sbk}\end{array}$ & 2 FI & $2 \mathrm{~F}$ & $5 Y 3 / 1$ & $\begin{array}{c}2 \%, \mathrm{FD}, 10 \mathrm{YR} 5 / 6, \mathrm{Fe} \\
\text { depletion }\end{array}$ \\
\hline Btg1 & $38-45$ & Massive & $2 \mathrm{FI}$ & $1 \mathrm{~F}+\mathrm{M}$ & $5 \mathrm{Y} 4 / 1$ & $5-10 \%, \mathrm{CP}, 10 \mathrm{YR} 4 / 6$ \\
\hline Btg2 & $45-70$ & Massive & $2 \mathrm{~F}+\mathrm{VF}$ & $1 \mathrm{~F}+\mathrm{M}$ & $\begin{array}{c}\text { 5Y4/1 (35\%) } \\
\text { 10YR 5/6 (65\%) }\end{array}$ & $2-5 \%, \mathrm{MD}, 7.5 \mathrm{YR} 4 / 4$ \\
\hline $\mathrm{Bg}$ & $70-105$ & Massive & - & $1 \mathrm{M}$ & $5 \mathrm{Y} 4 / 1$ & Gleying, Fe depletion \\
\hline \multicolumn{7}{|c|}{ P3- Paddy $\left(36^{\circ} 51^{\prime} 51.9^{\prime \prime} \mathrm{N} 50^{\circ} 46^{\prime} 51 \mathrm{E}\right)$} \\
\hline Apg & $0-12$ & Massive & $2 \mathrm{~F}+\mathrm{VF}$ & $2 \mathrm{~F}+\mathrm{VF}$ & $2.5 Y 3 / 2$ & $2 \%, \mathrm{FFa}$ \\
\hline $\mathrm{Bg}$ & $12-25$ & Massive & $1 \mathrm{VF}$ & $2 \mathrm{~F}+\mathrm{VF}$ & $5 \mathrm{Y} 4 / 1$ & $5-10 \%, \mathrm{MD}, 7.5 \mathrm{YR} 5 / 6$ \\
\hline
\end{tabular}




\begin{tabular}{|c|c|c|c|c|c|c|}
\hline Horizon & Depth & Structure $^{1}$ & Void $^{2}$ & $\operatorname{Root}^{3}$ & Color & $\begin{array}{l}\text { Redoximorphic } \\
\text { properties }^{4}\end{array}$ \\
\hline Btg1 & $25-60$ & sbk & - & $\begin{array}{l}2 \mathrm{~F} \\
\text { (rotten } \\
\text { root) }\end{array}$ & $\begin{array}{c}5 \text { Y } 4 / 1(70 \%) \\
10 \text { YR 5/6 (30\%) }\end{array}$ & $10-20 \%, \mathrm{MD}, 7.5 \mathrm{YR} 5 / 6$ \\
\hline Btg2 & $60-100$ & Massive & - & - & $5 \mathrm{Y} 4 / 1$ & Gleying \\
\hline \multicolumn{7}{|c|}{ P4- Paddy $\left(36^{\circ} 51^{\prime} 52.7^{\prime \prime} \mathrm{N} 50^{\circ} 46^{\prime} 48.9^{\prime \prime} \mathrm{E}\right)$} \\
\hline Apg & $0-20$ & Massive & $1 \mathrm{VFI}$ & $2 \mathrm{VF}+\mathrm{F}$ & $10 Y R 3 / 6$ & $2 \%, \mathrm{FD}$ \\
\hline 2Bg1 & $20-40$ & Massive & $2 \mathrm{VFI}$ & $2 \mathrm{~F}+\mathrm{VF}$ & $5 \mathrm{Y} 3 / 1$ & $2-5 \%, M D$ \\
\hline 2Bg2 & $40-75$ & Massive & 3 VFI & - & $5 \mathrm{Y} 4 / 1$ & Gleying \\
\hline \multicolumn{7}{|c|}{ P5- Paddy $\left(36^{\circ} 51^{\prime} 48^{\prime \prime} \mathrm{N} 50^{\circ} 46^{\prime} 49.5^{\prime \prime} \mathrm{E}\right)$} \\
\hline Apg & $0-14$ & Massive & $2 \mathrm{VFI}$ & $\begin{array}{c}\mathrm{VF} \\
+\mathrm{F}+\mathrm{M}\end{array}$ & 10YR 3/1 & - \\
\hline Btg1 & $14-35$ & $\begin{array}{l}1 \mathrm{~m} \mathrm{sbk} \\
1 \mathrm{~m} \mathrm{abk}\end{array}$ & $1 \mathrm{VF}$ & $1 \mathrm{VF}+1 \mathrm{M}$ & $\begin{array}{l}10 \text { YR 4/4 (75\%) } \\
10 \text { YR 5/1 }(25 \%)\end{array}$ & - \\
\hline Btg2 & $35-50$ & $\begin{array}{l}1 \mathrm{~m} \mathrm{sbk} \\
1 \mathrm{~m} \mathrm{abk}\end{array}$ & $1 \mathrm{VF}$ & $1 \mathrm{M}+1 \mathrm{C}$ & $\begin{array}{l}\text { 10YR 5/4 }(60 \%) \\
10 \text { YR 5/1 }(40 \%)\end{array}$ & - \\
\hline 2Bg1 & $50-75$ & $\mathrm{Sg}$ & $2 \mathrm{VFI}$ & rotten root & 2.5 Y $3 / 0$ & Gleying \\
\hline $3 \mathrm{Bg} 2$ & $75-110$ & Massive & 2 VFI & - & $2.5 \mathrm{Y} 4 / 0$ & Gleying \\
\hline \multicolumn{7}{|c|}{ P6- Paddy $\left(36^{\circ} 51^{\prime} 49.9^{\prime \prime} \mathrm{N} 50^{\circ} 46^{\prime} 46.1^{\prime \prime} \mathrm{E}\right)$} \\
\hline Apg & $0-16$ & Massive & - & $2 \mathrm{VF}+\mathrm{F}$ & $10 \mathrm{YR} 3 / 1$ & - \\
\hline $\mathrm{Bg} 1$ & $16-40$ & Massive & - & - & $2.5 \mathrm{Y} 3 / 1$ & $2-5 \%, \mathrm{FD}$ \\
\hline $\mathrm{Bg} 2$ & $40-100$ & Massive & - & - & $2.5 \mathrm{Y} 3 / 1$ & Gleying \\
\hline \multicolumn{7}{|c|}{ P7- Kiwi $\left(36^{\circ} 51^{\prime} 50.8^{\prime \prime} \mathrm{N} 50^{\circ} 46^{\prime} 44.5^{\prime \prime} \mathrm{E}\right)$} \\
\hline Ap & $0-12$ & Massive & $1 \mathrm{VF}+1 \mathrm{~F}$ & $1 \mathrm{~F}+1 \mathrm{VF}$ & 10YR 3/1 & - \\
\hline $\mathrm{Bg} 1$ & $12-27$ & $1 \mathrm{~m} \mathrm{sbk}$ & $1 \mathrm{VF}+1 \mathrm{~F}$ & - & $2.5 \mathrm{Y} 2 / 0$ & $5 \%, \mathrm{M}-\mathrm{CD}, 10 \mathrm{YR} 4 / 4$ \\
\hline $\mathrm{Bg} 2$ & $27-45$ & $1 \mathrm{~m} \mathrm{sbk}$ & $2 \mathrm{M}, \mathrm{C}$ & $\begin{array}{c}3 \mathrm{C}+3 \mathrm{M} \\
\text { Drastic } \\
\text { rotten root }\end{array}$ & $10 \mathrm{YR} 3 / 2$ & $5-10 \%, \mathrm{MD}$ \\
\hline $2 \mathrm{Cg}$ & $45-100$ & $\mathrm{Sg}$ & - & $\begin{array}{c}\text { Drastic } \\
\text { rotten root }\end{array}$ & 10YR 4/1 & - \\
\hline \multicolumn{7}{|c|}{ P8- Kiwi $\left(36^{\circ} 51^{\prime} 46.3^{\prime \prime} \mathrm{N} 50^{\circ} 46^{\prime} 53.3^{\prime \prime} \mathrm{E}\right)$} \\
\hline Apg & $0-12$ & Massive & $1 \mathrm{VF}$ & $\begin{array}{l}2 \mathrm{~F}+\mathrm{VF} \\
2 \mathrm{M}+2 \mathrm{C}\end{array}$ & $7.5 \mathrm{YR} 3 / 3$ & - \\
\hline $\mathrm{Bg}$ & $12-23$ & $1 \mathrm{~m} \mathrm{sbk}$ & $1 \mathrm{VF}$ & $\begin{array}{c}2 \mathrm{~F}+\mathrm{VF} \\
2 \mathrm{C}\end{array}$ & $7.5 \mathrm{YR} 4 / 3$ & $5-10 \%, \mathrm{MD}$ \\
\hline Btg1 & $23-45$ & $1 \mathrm{~m} \mathrm{sbk}$ & $2 \mathrm{M}+2 \mathrm{C}$ & $2 \mathrm{C}+2 \mathrm{M}$ & $7.5 \mathrm{YR} 3 / 5$ & $10-20 \%, \mathrm{MD}, 7.5 \mathrm{YR} 5 / 6$ \\
\hline Btg2 & $45-75$ & Massive & $2 \mathrm{M}+2 \mathrm{C}$ & $1 \mathrm{C}+1 \mathrm{M}$ & $7.5 \mathrm{YR} 4 / 0$ & $\begin{array}{c}2 \%, \mathrm{MD}, 7.5 \mathrm{YR} 5 / 6, \mathrm{Fe} \\
\text { depletion }\end{array}$ \\
\hline
\end{tabular}

1-Structure: Grade- 2: moderate, 1: weak. Size- m: medium. Type- Sg: single grain, abk: angular blocky, sbk: subangular blocky.

2-Void: abundance-1:very low( $<2 \%), 2: \operatorname{low}(2-5 \%), 3$ :medium(5-15\%). Type- I: interstitial. Size- VF:very fine, F:fine, M:medium, C:coarse

3-Root: abundance-1:few, 2:common, 3:many. Size- VF:very fine, F:fine, M:medium, C:coarse 4-Redoximorphicfeatures: abundance- M:many $(>20 \%)$, C:common(2-20\%), F: few $(<2 \%)$. Contrast- F:faint, D: distinct, P:prominent. 
Paddy soils pedons were not saturated all of the year and the water table fluctuation was

influenced by cropping and flooding of the rice growing and rainfall. Simultaneous surface waterlogging for rice cultivation in combination with high ground water table have resulted specific characteristics in the paddy soils. Aquic conditions in soils are often associated with redoximorphic features. Redoximorphic features such as $\mathrm{Fe}$ and Mn oxides. Color patterns and redoximorphic features of stagnic and reductive soils are the most significant characteristics of soils affected by water table depth and its fluctuation in studied soils (Table 1). The ground water table in the paddy soils mostly below $50 \mathrm{~cm}$ whereas the recorded depth in the non-paddy soil was at depths greater than $100 \mathrm{~cm}$. Under oxidative conditions existed in soils, $\mathrm{Fe}$ and $\mathrm{Mn}$ concretions (2-20\%) were observed as void coating on root channels, macropores and soil matrix. A reductive matrix (2.5Y2/0 to 10YR5/6) associated with Fe-depleted or enriched features (Table 1) were taken as evidence for water table fluctuations (paddy soils pedons). Soil organic carbon content was relatively high in the surface horizons of paddy soils due to incorporation of crop litter and rotten roots caused by very poor drainage, and hence lower rate of biological activities and also lowers organic matter decomposition.

Result showed that $\mathrm{pH}_{\mathrm{sp}}$ varies between 7.8 and 8.3 for paddy soils, and between 7.3 and 8.4 for non- paddy soils (Table 2). Electrical conductivity of saturation extracts (ECe) was between 336 and $2130 \mu \mathrm{S}$ $\mathrm{m}^{-1}$. Relatively strong differences $\left(0.2\right.$ to $\left.6.7 \mathrm{~g} \mathrm{~kg}^{-1}\right)$ were observed in the horizontal (between pedons) and vertical (between horizons) distribution of soils' organic carbon. The largest amount of organic carbon was observed in the surface layer of the kiwi-fruit soils (pedons 7 and 8) due to higher accumulation of the surface litter and addition of rotted roots. Cation exchange capacities (CEC) were measured between 4.8 (pedon 1) to 36.2 (pedon 7) $\mathrm{cmol}^{+} \mathrm{kg}^{-1}$. The lowest $\mathrm{CEC}$ was obtained in the deeper horizons of the citrus pedon, due to their lower clay and organic-matter contents. In all of the studied pedons, the highest CEC values were found in the surface layers as a result of their higher organic carbon and clay contents. The paddy soils had the highest clay content among the studied pedons. The calcium carbonate equivalent (CCE) in paddy soil and citrus pedons was between trace to $20.8 \%$ (Table 2). The highest water-soluble $\mathrm{K}$ content was observed in the citrus pedons $(17.9 \mathrm{mg}$ $\left.\mathrm{kg}^{-1}\right)$, followed by kiwi fruit $\left(10.5 \mathrm{mg} \mathrm{kg}^{-1}\right)$ and paddy soils (3.1 mg kg-1). In contrast, the highest content of exchangeable $\mathrm{K}$ was observed in the kiwi-fruit pedons (165 $\left.\mathrm{mg} \mathrm{kg}^{-1}\right)$, followed by citrus $\left(121 \mathrm{mg} \mathrm{kg}^{-1}\right)$ and paddy soil $\left(82 \mathrm{mg} \mathrm{kg}^{-1}\right)$. For non-exchangeable $\mathrm{K}$, the paddy soil pedon had the highest value $\left(697 \mathrm{mg} \mathrm{kg}^{-1}\right)$, followed by citrus $\left(642 \mathrm{mg} \mathrm{kg}^{-1}\right)$ and kiwi fruit (281 $\mathrm{mg} \mathrm{kg}^{-1}$ ) (Table 2). 
Table 2. Land use, soil classification and some physico-chemical properties of the studied pedons.

\begin{tabular}{|c|c|c|c|c|c|c|c|c|c|c|c|c|c|}
\hline Horizon & PSD & (g kg & $\left.r^{-1}\right) *$ & Tex.* & $\mathbf{p H}_{\mathrm{sp}}$ & EC & $\mathrm{OC}^{*}$ & CEC* & $\begin{array}{l}\text { CEC/ } \\
\text { Clay }\end{array}$ & $\mathrm{CCE}^{*}$ & $\mathbf{K}_{\text {sol }}{ }^{*}$ & $\mathbf{K}_{\mathrm{ex}}^{*}$ & $\mathbf{K}_{\text {nonex }}{ }^{*}$ \\
\hline & Sand & Silt & Clay & & & $\mu \mathrm{S} \mathrm{m} \mathbf{m}^{-1}$ & $\mathbf{g ~ k g}^{-1}$ & $\mathrm{cmol}^{+} \mathbf{k g}^{-1}$ & $\%$ & $\%$ & \multicolumn{3}{|c|}{$\mathrm{mg} \mathrm{kg}^{-1}$} \\
\hline \multicolumn{14}{|c|}{ (P1)Citrus: Sandy, vermiculitic, thermic, Oxiaquic Udipsamments } \\
\hline Ap & 72.4 & 12 & 15.6 & SL & 7.8 & 1022 & 1.9 & 15.2 & 0.9 & Trace & 18 & 121 & 642 \\
\hline $\mathrm{AC}$ & 86.4 & 4 & 9.6 & LS & 8.1 & 557 & 0.4 & 7.9 & 0.8 & 2.9 & 4.3 & 35 & 335 \\
\hline $\mathrm{C} 1$ & 90.4 & 4 & 5.6 & $\mathrm{~S}$ & 8.1 & 347 & 0.2 & 4.7 & 0.8 & 4.9 & 2.1 & 30 & 260 \\
\hline $\mathrm{C} 2$ & 92.4 & 4 & 3.6 & $\mathrm{~S}$ & 8.4 & 336 & 0.2 & 4.9 & 1.4 & 4.1 & 2.5 & 26 & 284 \\
\hline \multicolumn{14}{|c|}{ (P2) Paddy: Fine-loamy, mixed, active, thermic, Typic Endoaqualfs } \\
\hline Apg & 17.8 & 30 & 52.2 & $\mathrm{C}$ & 7.8 & 1261 & 4.7 & 28.9 & 0.5 & Trace & 3.1 & - & - \\
\hline $\mathrm{Bg}$ & 12.4 & 30 & 57.6 & $\mathrm{C}$ & 7.8 & 842 & 2.2 & 30.2 & 0.5 & Trace & 1.6 & - & - \\
\hline Btg1 & 14.4 & 32 & 53.6 & $\mathrm{C}$ & 8.1 & 559 & 2.2 & 22.3 & 0.4 & 2.6 & 1.9 & - & - \\
\hline Btg2 & 36.4 & 36 & 27.6 & $\mathrm{CL}$ & 8.3 & 421 & 0.6 & 14.9 & 0.5 & 2.8 & 0.9 & - & - \\
\hline $\mathrm{Bg}$ & 20.4 & 48 & 31.6 & $\mathrm{CL}$ & 8.3 & 399 & 0.6 & 17.2 & 0.5 & 7.1 & 1.6 & - & - \\
\hline \multicolumn{14}{|c|}{ (P3) Paddy: Fine, smectitic, thermic, Typic Endoaqualfs } \\
\hline Apg & 23.1 & 27 & 49.9 & $\mathrm{C}$ & 7.8 & 1030 & 3.7 & 21.6 & 0.4 & 8.7 & 1.9 & 58 & 253 \\
\hline $\mathrm{Bg}$ & 22.4 & 34 & 43.6 & $\mathrm{C}$ & 7.9 & 1022 & 3.7 & 25.1 & 0.6 & 8.6 & 1.9 & 65 & 277 \\
\hline Btg1 & 12.4 & 42 & 45.6 & $\mathrm{SiC}$ & 8.2 & 563 & 1.9 & 21.3 & 0.5 & 20.8 & 1.6 & 51 & 697 \\
\hline Btg2 & 10.4 & 46 & 43.6 & $\mathrm{SiC}$ & 8.1 & 524 & 0.6 & 18.1 & 0.4 & 3.4 & 1.6 & 52 & 189 \\
\hline \multicolumn{14}{|c|}{ (P4) Paddy: Fine-loamy, smectitic, thermic, Typic Endoaquepts } \\
\hline Apg & 29.4 & 27 & 43.6 & $\mathrm{C}$ & 7.8 & 1095 & 0.8 & 21.1 & 0.5 & 7.9 & 2.7 & 55 & 190 \\
\hline $2 \mathrm{Bg} 1$ & 58.4 & 26 & 15.6 & SL & 7.9 & 1119 & 0.2 & 9.6 & 0.6 & 1.9 & 1.9 & 25 & 198 \\
\hline 2Bg2 & 26.4 & 46 & 27.6 & $\mathrm{CL}$ & 7.9 & 866 & 0.6 & 12.3 & 0.4 & 7.9 & 2.3 & 51 & 140 \\
\hline \multicolumn{14}{|c|}{ (P5) Paddy: Fine, mixed, active, thermic, Typic Endoaqualfs } \\
\hline Apg & 17.8 & 30 & 52.2 & $\mathrm{C}$ & 7.8 & 1055 & 2.6 & 24.8 & 0.5 & 1.4 & 2.3 & 82 & 299 \\
\hline Btg1 & 10.4 & 36 & 53.6 & $\mathrm{C}$ & 8.1 & 716 & 1.4 & 24.1 & 0.4 & 0.3 & 1.6 & 69 & 313 \\
\hline Btg2 & 16.4 & 40 & 43.6 & $\mathrm{C}$ & 8.2 & 501 & 0.9 & 18.8 & 0.4 & 1.9 & 2.7 & 60 & 229 \\
\hline $2 \mathrm{Bg} 1$ & 56.4 & 26 & 17.6 & $\mathrm{SL}$ & 7.9 & 917 & 0.4 & 9.6 & 0.5 & 1.3 & 2.1 & 30 & 180 \\
\hline $3 \mathrm{Bg} 2$ & 26.4 & 46 & 27.6 & L & 7.9 & 841 & 0.9 & 12.1 & 0.4 & 9.1 & 2.3 & 45 & 209 \\
\hline \multicolumn{14}{|c|}{ (P6) Paddy: Fine, mixed, superactive, thermic, Typic Endoaquepts } \\
\hline Apg & 10.5 & 36 & 53.5 & $\mathrm{C}$ & 7.7 & 1263 & 4.5 & 24.8 & 0.5 & 4.2 & 2.7 & - & - \\
\hline Bg1 & 32.4 & 34 & 33.6 & $\mathrm{CL}$ & 7.7 & 1163 & 2.8 & 24.7 & 0.7 & 2.2 & 2.3 & - & - \\
\hline $\mathrm{Bg} 2$ & 30.4 & 34 & 35.6 & $\mathrm{CL}$ & 7.7 & 1113 & 3.2 & 23.4 & 0.7 & 9.6 & 2.3 & - & - \\
\hline \multicolumn{14}{|c|}{ (P7) Kiwi: Fine-loamy, mixed, superactive, thermic, Typic Endoaquepts } \\
\hline $\mathrm{Ap}$ & 31.8 & 26 & 42.2 & $\mathrm{C}$ & 7.7 & 1005 & 6.7 & 30.4 & 0.7 & Trace & 6.6 & 111 & 281 \\
\hline $\mathrm{Bg} 1$ & 32.4 & 32 & 35.6 & $\mathrm{CL}$ & 7.6 & 882 & 5.3 & 32.6 & 0.9 & Trace & 3.1 & 56 & 247 \\
\hline $\mathrm{Bg} 2$ & 38.4 & 32 & 29.6 & $\mathrm{CL}$ & 7.3 & 1459 & 5.9 & 36.2 & 1.2 & Trace & 3.1 & 41 & 175 \\
\hline $2 \mathrm{Cg}$ & 52.4 & 28 & 19.6 & SL & 7.3 & 2130 & 2.8 & 17.2 & 0.9 & Trace & 3.5 & 30 & 207 \\
\hline \multicolumn{14}{|c|}{ (P8) Kiwi: Fine-loamy, vermiculitic, thermic, Oxiaquic Hapludalfs } \\
\hline Apg & 8.5 & 29 & 62.5 & $\mathrm{C}$ & 7.6 & 1066 & 5.3 & 34.1 & 0.5 & Trace & 10.5 & 165 & 268 \\
\hline $\mathrm{Bg}$ & 24.4 & 32 & 43.6 & $\mathrm{C}$ & 8.1 & 587 & 0.9 & 24.2 & 0.6 & Trace & 1.9 & 62 & 202 \\
\hline Btg1 & 12.4 & 44 & 43.6 & $\mathrm{SiC}$ & 8.4 & 494 & 0.9 & 23.2 & 0.5 & Trace & 1.6 & 50 & 218 \\
\hline Btg2 & 22.4 & 46 & 31.6 & CL & 8.3 & 578 & 0.6 & 18.2 & 0.6 & Trace & 2.3 & 55 & 217 \\
\hline
\end{tabular}

*PSD: Particle size distribution, OC: organic carbon, CEC: cation exchange capacity, CCE: Calcium carbonate equivalent, $\mathrm{K}_{\text {sol }}$ : Water soluble potassium, $\mathrm{K}_{\mathrm{ex}}$ : Exchangable potassium, $\mathrm{K}_{\text {nonex }}$ :Nonexchangable potassium, SL: sandy loam, LS: loamy sand, S: sand, C: clay, CL: clay loam, L: loam, SiC: silty clay. 
The mineral composition of studied soils is shown by the X-ray diffractograms of the clay fraction (Figure 2 and Table 3). The high intensity of 1.4 and $0.7 \mathrm{~nm}$ peaks in $\mathrm{Mg}$-saturated $(\mathrm{Mg})$ and $\mathrm{Mg}$-saturated, glycerol-solvated treatments $(\mathrm{Mgg})$, and the collapse of the peaks in K-saturated (K) and K550 (KT) treatments demonstrate the presence of vermiculite (Figure 2D). Also, $1,0.5$ and $0.33 \mathrm{~nm}$ peaks in $\mathrm{Mg}$-saturated diffractograms indicate the presence of illite. Kaolinite was detected based on the presence of 0.7 and 3.56 $\mathrm{nm}$ peaks in $\mathrm{Mg}$-saturated treatment that collapsed in KT treatment. A shifting of the $1.4 \mathrm{~nm}$ peak in the Mgsaturated treatment toward $1.8 \mathrm{~nm}$ in $\mathrm{Mg}$ saturated-gly- cerol solvated treatment also indicated the presence of some expandable clay minerals (smectites) (Figure 2A and B). A shifting of the $1.4 \mathrm{~nm}$ peak towards 1.1-1.3 $\mathrm{nm}$ in KT treatment showed the presence of hydroxyinterlayered minerals. Vermiculite, smectite and vermiculite, hydroxy-interlayered vermiculite were the most prevailing clay minerals in citrus, paddy and kiwi fruit pedons, respectively (Table 3). Differences in clay mineralogical suites were most probably related to land management, parent materials (lacustrine deposits under citrus and fine to coarse layered alluvium under paddy and kiwi pedons) and soil-drainage conditions (by controlling the stability of minerals).
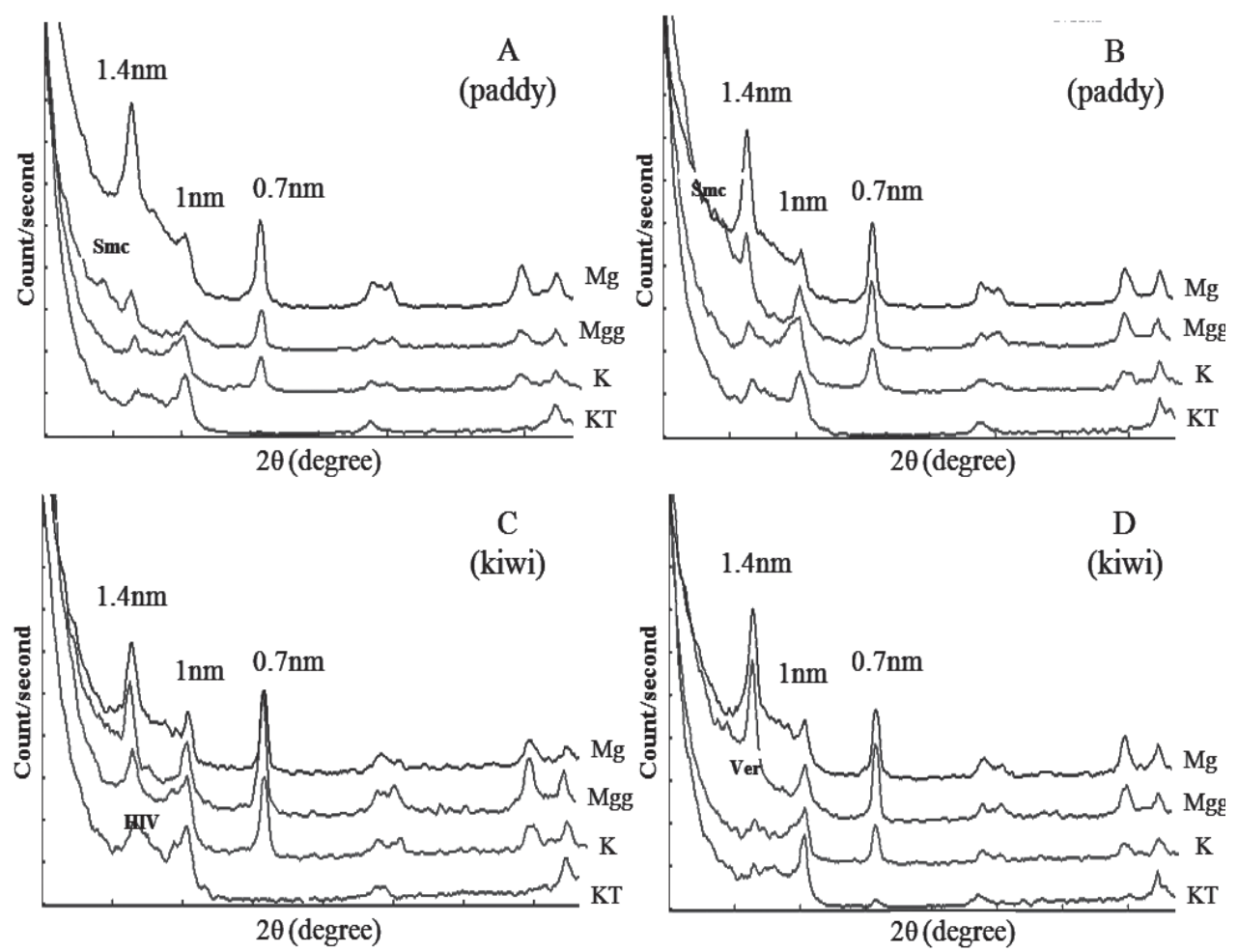

Figure 2. Representative X-ray diffractograms: A: 2Bg2 horizon, pedon 4, B: 3Bg2 horizon, pedon 5, C: $\mathrm{Bg} 2$ horizon, pedon 7, D: Btg1 horizon, pedon 8. 
Table 3. Clay mineralogical suite of the some studied soil samples.

\begin{tabular}{|c|c|c|c|}
\hline Horizon & Mineralogy & Horizon & Mineralogy \\
\hline \multicolumn{2}{|r|}{ (P3) Paddy } & \multicolumn{2}{|r|}{ (P7) kiwi } \\
\hline Apg & Illi. $>$ HIV. $>$ Smc. $>$ Kao. $>$ Ver. & Ap & Smc. $>$ Ver. $>$ Illi. $>$ Kao. $>$ HIV. \\
\hline $\mathrm{Bg}$ & $>$ Kao. Smc. $>$ Ver. $>$ HIV. $>$ Illi. & $\mathrm{Bg} 1$ & HIV. $>$ Smc. $>$ Ver. $>$ Illi. $>$ Kao. \\
\hline Btg1 & Illi. $>$ HIV. $>$ Ver. $>$ Kao. $>$ Smc. & $\mathrm{Bg} 2$ & HIV. $>$ Smc. $>$ Ver. $>$ Illi. $>$ Kao. \\
\hline Btg2 & Illi. $>$ HIV. $>$ Ver. $>$ Kao. $>$ Smc. & $2 \mathrm{Cg}$ & HIV. $>$ Smc. $>$ Ver. $>$ Illi. $>$ Kao. \\
\hline \multicolumn{2}{|r|}{ (P4) Paddy } & \multicolumn{2}{|r|}{ (P8) kiwi } \\
\hline Apg & Smc. $>$ Illi. $>$ Kao. $>$ HIV. $>$ Ver. & Apg & Smc. $>$ HIV. $>$ Ver. $>$ Illi. $>$ Kao. \\
\hline 2Bg1 & Smc. $>$ Ver. $>$ Illi. $>$ Kao. $>$ HIV. & $\mathrm{Bg}$ & Ver. $>$ HIV-Cl. > Kao. > Illi. > Smc. \\
\hline $2 \mathrm{Bg} 2$ & Smc. $>$ Illi. $>$ Kao. $>$ HIV. $>$ Ver. & Btg1 & Ver. $>$ HIV-Cl. > Kao. > Illi. > Smc. \\
\hline \multicolumn{2}{|r|}{ (P5) Paddy } & Btg2 & Ver. $>$ Smc. $>$ HIV-Cl. $>$ Kao. > Illi. \\
\hline Apg & $>$ Smc. Ver. $>$ Illi. $>$ HIV. $>$ Kao & \multicolumn{2}{|r|}{ (P1) Citrus } \\
\hline Btg1 & HIV. $>$ Kao. $>$ Illi. $>$ Ver. $>$ Smc. & Ap & Ver. $>$ Smc. $>$ Mix. $>$ HIV. $>$ Illi. $>$ Kao. \\
\hline Btg2 & Smc. $>$ HIV. $>$ Ver. $>$ Illi. $>$ Kao. & $\mathrm{AC}$ & $>$ Illi. Ver. $>$ Smc. $>$ HIV. $>$ Kao \\
\hline 2Bg1 & Smc. $>$ HIV. $>$ Ver. $>$ Illi. $>$ Kao. & $\mathrm{C} 1$ & $>$ Smc. Ver. $>$ Illi. $>$ HIV. $>$ Kao. \\
\hline $3 \mathrm{Bg} 2$ & Smc. $>$ Illi. $>$ Ver. $>$ Kao. $>$ HIV. & $\mathrm{C} 2$ & $>$ Smc. Ver. $>$ Illi. $>$ HIV. $>$ Kao. \\
\hline
\end{tabular}

* Illi.: Illite, HI.: Hydroxy-Interlayere Vermiculite, Smc.: Smectite, Kao.: Kaolinite, Ver.: Vermiculite, Cl.: Clorite, Mix.: Mixed mineral.

\section{Discussion}

Clay mineralogy is probably more influenced by aquic and anthraquic conditions. Also, there are some indications that aquic and anthraquic conditions may affect on quantity of clay minerals, as shown by higher smectite in poorly drained soils. Clay minerals transformations are strongly dependent on $\mathrm{K}$ amounts in soil and also the dynamics either causing release or removal of $\mathrm{K}$ to or from the soil. Mineralogical results indicated that smectite was the most dominant mineral in the studied paddy soils whereas in non-paddy soils with moderately drainage conditions, smectite content decreased and vermiculite content increased. In non-paddy soils, potassium fertilization and irrigation during the growing season moves potassium to the deeper soil (below $12 \mathrm{~cm}$ ) and causes changes in clay minerals from smectite to vermiculite. Liu et al. (2007) stated that the longterm use of potassium fertilizers in the soil increased the amount of illite and vermiculite due to specific $\mathrm{K}$ fixation in the wedge zones caused by interactions between clay minerals and $\mathrm{K}$ presence. The complexity of dominant conditions affecting mineralogical composition of the studied paddy soils (e.g. surface flooding, high ground water table, lithological discontinuities and limited potassium fertilization) have caused problems in interpretation of the clay mineralogical variations between pedons and horizons in each pedon. However, in the deeper soils' horizons, smectite dominated and in the surface horizon, illite, vermiculite and HIV dominated. In addition, appropriate conditions (i.e. high $\mathrm{Si}, \mathrm{Mg}$, and suitable $\mathrm{pH}$ ) provided by poor drainage have led to the stability 
of smectitic minerals. Smectite is the most prevalent minerals in aquic soils and under very poor drainage conditions such as in rice paddy soils (Liu et al., 2007; Zhang et al., 2011).

The exchangeable and non-exchangeable potassium in the surface layers of the citrus pedons were higher than that in the subsurface layers due to more suitable thermal and moisture status preconditioned for mica and feldspar weathering (Figure 3A). K does not leach from clayey and silty soils, but the sandy soil used for citrus is not able to hold high amounts of exchangeable and non-exchangeable potassium. Nabiollahy et al. (2006) reported that mineral K and
HNO3-extractable $\mathrm{K}$ in the soils containing less illite (10-30\%) were significantly different from those with more illite (30-50\%).

Besides the clay content, K-bearing minerals and clay mineralogy may play a more important role in determining a soil's extractable K status. For example, the clay contents in pedon 1 (citrus) were relatively low, and their dominant clay mineral was vermiculite, whereas the measured exchangeable and non-exchangeable $\mathrm{K}$ was medium and high (Towfighi, 1998), respectively (Table 2). Wedge zones or specific K-adsorption sites in vermiculite may have caused these results (Barré et al., 2007).

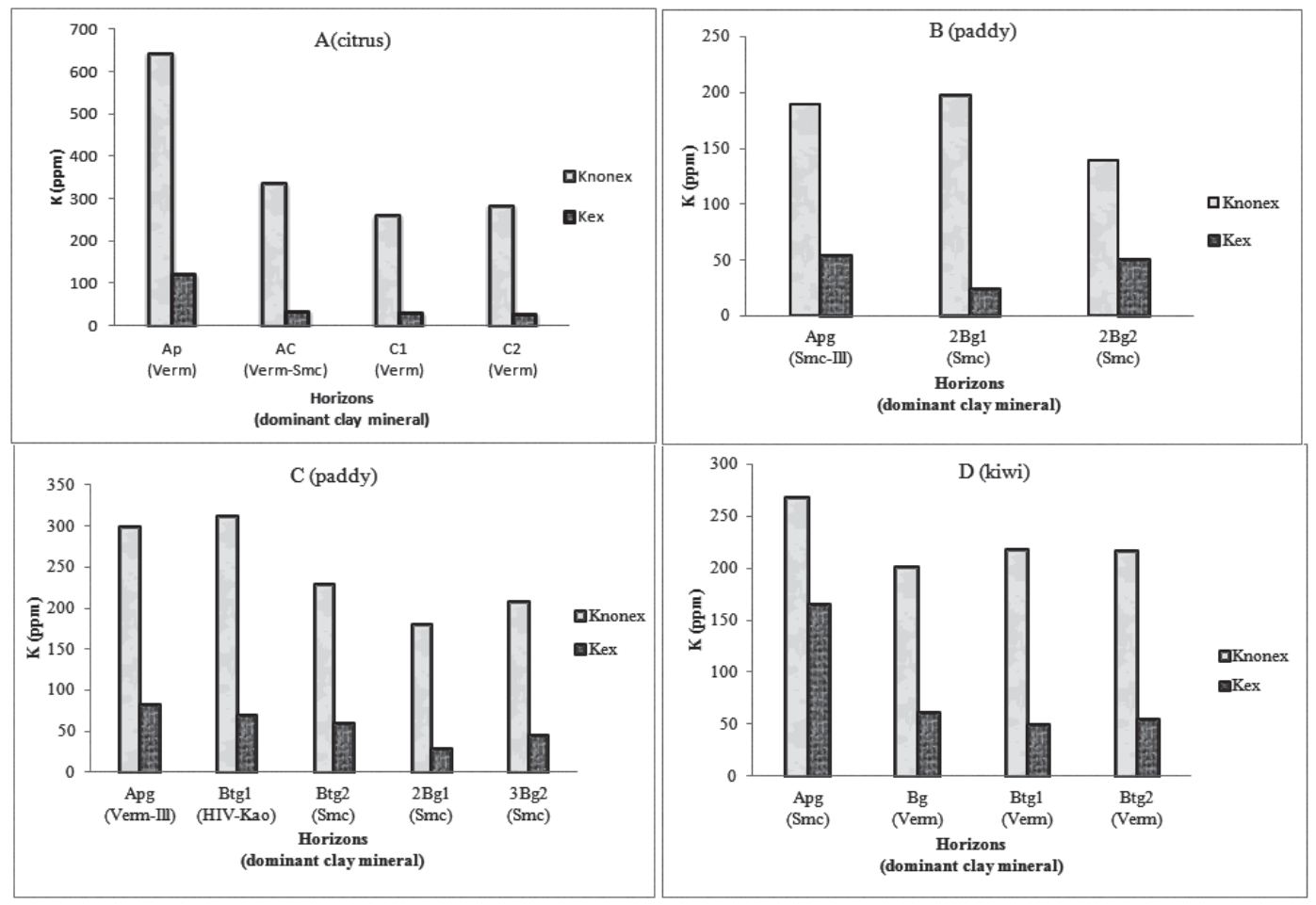

Figure 3. Relationship between dominant clay minerals with exchangeable and non-exchangeable potassium in different horizons, A: pedon 1(citrus), B: pedon 4 (paddy), C: pedon 5 (paddy), D: pedon 8 (kiwi). 
Plant-available potassium (soluble K) and exchangeable potassium in the studied soils were very low (Table 2). Mean soluble and exchangeable potassium in paddy soil samples were lower than in kiwi and citrus soil samples. Smectite was the most prevalent mineral in the paddy soils; this may be the main reason for their less available $\mathrm{K}$ forms compared to other land uses. K uptake decreases as soil moisture content increases to saturation. Shahbazi and Towfighi (2006) showed that exchangeable $\mathrm{K}$ decreases with increasing soil-saturation time.

Results in this study showed that in soil samples in which smectite predominated, non-exchangeable potassium was about 200-300 $\mathrm{m} \mathrm{kg}^{-1}$, while in vermiculite and hydroxy-interlayered vermiculite and illitedominated soil samples, non-exchangeable potassium was about 300-600 mg kg-1 (Figure 3). Illitic soils, due to the higher fixed potassium in their interlayer positions, and vermiculitic soils, due to their morenegative layer charge, are among the most important factors in controlling a soils' potassium status. Sparks (1987) and Moritsuka et al. (2004) reported that the major sources of non-exchangeable $\mathrm{K}$ in soils are $\mathrm{K}$ rich 2:1 clay minerals such as micas and vermiculite.

The correlation between physico-chemical properties such as clay content, organic carbon, CEC and calcium carbonate equivalent with different forms of potassium for three groups of soils are given in Ta- ble 4. Soluble form of potassium showed significant and positive correlation with exchangeable and nonexchangeable forms but the relation between exchangeable and non-exchangeable $\mathrm{K}$ was not significant $\left(0.50^{*}-0.72^{* *}-0.32^{\mathrm{n}}\right)$. On the other hand, with increasing content of one or two forms of $\mathrm{K}$, the content of other forms of $\mathrm{K}$ were increased (Table 4).

The results show that the clay content, organic carbon and cation exchange capacity are significantly correlated with exchangeable potassium $\left(0.56^{* *}, 0.54^{* *}, 0.56^{* *}\right.$, respectively) while other forms of potassium do not show a noticeable correlation. The high correlation between exchangeable potassium with clay content and CEC refers especially to the clay surface which is able to adsorb and exchange potassium in the soil. Sinha and Biswas (2003) stated that water soluble, plant-available and non-exchangeable potassium showed a positive and significant correlation with clay content and cation exchange capacity for soils.

The calcium carbonate equivalent seems to be an important factor influencing all forms of $\mathrm{K}$ in studied soils. There are negative correlations between calcium carbonate content and all forms of potassium except for non-exchangeable K. Najafi Ghiri et al. (2010) found that calcium carbonate content had a negative effect on different soil $\mathrm{K}$ pools except for water soluble $\mathrm{K}$ which resulted from the greater leaching of $\mathrm{K}$. 
Table 4. Correlations between physico-chemical properties with potassium forms.

\begin{tabular}{lccccccc}
\hline & Clay & OC & CEC & CCE & $\mathbf{K}_{\text {sol }}$ & $\mathbf{K}_{\text {ex }}$ & $\mathbf{K}_{\text {nonex }}$ \\
\hline Clay & 1 & & & & & & \\
OC & $0.46^{* *}$ & 1 & & & & & \\
CEC & $0.77^{* *}$ & $0.81^{* *}$ & 1 & & & & \\
CCE & 0.00 & -0.16 & -0.22 & 1 & & & \\
$\mathrm{~K}_{\text {sol }}$ & -0.11 & 0.28 & 0.09 & -0.26 & 1 & & \\
$\mathrm{~K}_{\text {ex }}$ & $0.56^{* *}$ & $0.54^{* *}$ & $0.56^{* *}$ & -0.22 & $0.72^{* *}$ & 1 & \\
$\mathrm{~K}_{\text {nonex }}$ & 0.00 & 0.06 & -0.01 & $0.41^{*}$ & $0.50^{*}$ & 0.32 & 1 \\
\hline
\end{tabular}

**, * Correlation is significant at the 0.01 and 0.05 level, respectively.

OC: organic carbon, CEC: cation exchange capacity, CCE: calcium carbonate equivalent, $\mathrm{K}_{\text {sol }}$ : Water soluble potassium, $\mathrm{K}_{\mathrm{ex}}$ : Exchangable potassium, $\mathrm{K}_{\text {nonex }}$ : Nonexchangable potassium.

In general, paddy soils' mean non-exchangeable potassium content was between moderately low and low (according to classification by Towfighi, 1998). This may be attributed to the prevalence of smectite, which can cause these soils to be rich in exchangeable potassium, yet moderately poor in $\mathrm{HNO}_{3}-\mathrm{K}$ concentration. Years of cultivation without the use of potassium fertilizers (100-150 $\mathrm{kg} \mathrm{h}^{-1}$ for local rice) have led to a drastic decrease in exchangeable potassium, followed by a decrease in non-exchangeable potassium in paddy soils.

\section{Conclusion}

Exchangeable $\mathrm{K}\left(\mathrm{NH}_{4} \mathrm{OAc}\right.$-extractable $\left.\mathrm{K}\right)$ is used as an indicator for the availability of potassium in soils, but it does not give similar trends in all mineralogical suites. A combination of both $\mathrm{NH}_{4} \mathrm{OAc}-\mathrm{K}$ and $\mathrm{HNO}_{3}-\mathrm{K}$ (non-exchangeable $\mathrm{K}$ ) gives a more applicable index for smectitic, illitic and vermiculitic soils. This study found that while smectitic soils are rich in $\mathrm{NH}_{4} \mathrm{OAc}-\mathrm{K}$, they have modest amounts of $\mathrm{HNO}_{3}$ $\mathrm{K}$, with moderate $\mathrm{K}$ release rates. On the other hand, illitic soils have only modest amounts of $\mathrm{NH}_{4} \mathrm{OAc}-\mathrm{K}$ with high amounts of $\mathrm{HNO}_{3}-\mathrm{K}$, and high rates of $\mathrm{K}$ release. Accordingly, shallow smectitic soils could experience $\mathrm{K}$ exhaustion and the need for $\mathrm{K}$ fertilization more than illitic soils. Non-exchangeable K plays a significant role in supplying available $\mathrm{K}$, particularly in soils containing K-bearing minerals. Therefore determination of both exchangeable and non-exchangeable $\mathrm{K}$ gives a better indication of the $\mathrm{K}$ potential and management for rice production in paddy soils than single determination. Hence, clay mineralogy should be considered as a main determinant in paddy soils' nutrient status. To improve rice production and potassium reserves in these paddy soils precise potassium management is required. As the main conclusion, potassium status of the soils is greatly influenced by the type of clay minerals and their abundance in soils or in parent rock and some physico-chemical properties such as clay content, organic carbon, cation exchange capacity. So, for better understanding of the relationship between clay mineralogy and different forms of potassium, studying the processes and mechanisms of potassium absorption and release is essential to achieve sustainable agriculture and increase the efficiency of crop production. 


\section{Acknowledgment}

The financial support provided by the University of Tehran, Iran, is gratefully acknowledged.

\section{References}

Alfaro, M. A., Jarvis, S.C., Gregory, P. J. 2003. The effect of grassland soil managements on soil potassium availability. J. Soil Sci. Plant Nutr. 3, 2, $31-41$.

Barré, P., Velde, B., Catel, N., Abbadie, L. 2007. Soilplant potassium transfer: impact of plant activity on clay minerals as seen from X-ray diffraction. Plant Soil. 292, 137-146.

Bhonsle, N.S., Pal, S.K., Sekhon, G.S. 1992. Relationship of $\mathrm{K}$ forms and release characteristics with clay mineralogy. Geoderma. 54, 285-293.

Britzke, D., Da Silva, L.S., Moterle, D.F., Rheinheimer, D. D.S., Bortoluzzi, E. C. 2012. A study of potassium dynamics and mineralogy in soils from subtropical Brazilian lowlands. J. Soils Sediments. 12,185-197.

Carter, M. R., Gregorich, E. G. 2008. Soil Sampling and Methods of Analysis. $2^{\text {nd }}$ (ed). Can. Soc. Soil Sci. 1224 p.

Ghosh, B.N., Singh, R.D. 2001. Potassium release characteristics of some soils of Uttar Pradesh hills varying in altitude and their relationship with forms of soil $\mathrm{K}$ and clay mineralogy. Geoderma. 104, 135-144.

Grossman, R.B., Millet, J.L. 1961. Carbonate removal from soils by a modification of the acetate buffer method. Soil Sci. Soc. Am. J. 25, 325-326.

Huang, P. M. 2005. Chemistry of potassium in soils. In: M. A. Tabatabai, D. L. Sparks (eds.) Chemical Processes in Soils. Soil Science Society of America, Madison, WI, USA, pp: 227-292.
Knudsen, D., Peterson, G.A., Pratt, P.F. 1996. Lithium, Sodium and Potassium. In: A. L. Page (ed) Methods of Soil Analysis. Part 2, Second edition, American Society Agronomy, Madison, WI, pp: 403-429.

Kunze, G. W., Rich, C.I. 1959. Mineralogical methods. In: C. I. Rich, L. F. Satz, G. W. Kunze (eds) Certain properties of selected southestern United States soils and mineralogical procedures for the study. Southern Coop, Series Bul, pp: 135-146.

Kunze, G.W., Dixon, J.B. 1986. Method of soil Analysis, Part 1. Physical and Mineralogical Methods. Amrican Society Agronomy.

Kyuma, K. 2004. Paddy soil science. Kyoto, Japan, Kyoto University Press, 280 p.

Liu, Y.L., Zhang, B., Cheng-liang, L., Feng H., Velde, B. 2007. Long term fertilization influences on clay mineral composition and ammonium adsorption in rice paddy soil. Soil Sci. Soc. Am. J. 72, 1580-1590.

Mehra, G.W., Jackson, M.L. 1960. Iron oxide removal from soils and clays by a dithionite-citrate system buffered with sodium bicarbonate. In: Clays and Clay Minerals, Proceeding, $7^{\text {th }}$ Conference, pp: 317-327.

Moritsuka, N., Yanai, J., Kosaki, T. 2004. Possible processes releasing non-exchangeable potassium from the rhizosphere of maize. Plant Soil. 258, 261-268.

Nabiollahy, K., Khormali, F., Bazargan, K., Ayoubi, Sh. 2006. Forms of $\mathrm{K}$ as a function of clay mineralogy and soil development. Clay. Clay Miner. 41, 739-749.

Najafi Ghiri, M., Abtahi, A., Jaberian, F., Owliaie, H.R. 2010. Relationship between Soil Potassium Forms and Mineralogy in Highly Calcareous Soils of Southern Iran. Aust. J. Basic \& Appl. Sci. $4,3,434-441$. 
Newhall, F., Berdanier, C. R. 1996. Calculation of soil moisture regimes from the climatic record. NRCS, Soil Survey Investigation Report, No. 46.

Pansu, M., Gautheyrou, J. 2006. Handbook of Soil Analysis: Mineralogical, Organic and Inorganic Methods. Springer. 995 p.

Pratt, P.F. 1965. Method of soil Analysis. American Society Agronomy. Madison, WI.

Rahimi Petroudi, E., Noormohammadi, Gh., Mirhadi, M.J., Madani, H., Mobasser, H.R. 2011. Effects of nitrogen fertilization and rice harvest height on agronomic yield indices of ratoon rice-berseem clover intercropping system. Aust. J. Crop Sci. 5, $5,566-574$.

Shahbazi, K., Towfighi, H. 2006. Effects of different moisture regimes on soil exchangeable potassium. Iran J. Agric. Sci. 38, 161-171.

Sharpley, N. 1989. Relationship between Soil Potassium Forms and Mineralogy. Soil Sci. Soc. Am. J. 52, 1023-1028.

Sinha, A.K., Biswas, S. 2003. Distribution of different forms of potassium in surface and subsurface horizons of some well established soils of West Bengal under the order Inceptisols. J. Interacademicia. 7 , 3, 286-291.

Soil Survey Staff. 2010. Keys to Soil Taxonomy, United States Department of Agriculture. 11nd (ed), NRCS.
Sparks, D.L. 1987. Potassium dynamics in soils. Adv. Soil Sci. 6, 1-63.

Srinivasarao, Ch., Rupab, T.R., Subba Rao, A., Ramesha, G., Bansald, S.K. 2006. Release Kinetics of Non-exchangeable Potassium by Different Extractants from Soils of Varying Mineralogy and Depth. Soil Sci. Plant Anal. 37,3, 473-491.

Surapaneni, A., Palmer, A.S., Tillman, R.W., Kirkman, J.H., Gregg, P.E.H. 2002. The mineralogy and potassium supplying power of some loessial and related soils of New Zealand. Geoderma. 110, 191-204.

Towfighi, H. 1998. Study of rice response to potassium fertilizer in paddy soils of northern Iran. Iranian J. Agric. Sci. 29, 869-883.

Witt, C., Haefele, S.M. 2005. Paddy soils. In: D. Hillel (ed), Encyclopedia of soils in the environment. Vol. 3. Academic Press; Columbia University, New York, USA. pp: 141-150.

Zhang, Q.C., Wang, G H., Feng, Y.K., Qian, P., Schoenau, J.J. 2011. Effect of potassium fertilization on soil potassium pools and rice response in an intensive cropping system in China. J. Plant Nutr. Soil Sci. 174, 73-80. 
